\title{
Expectation Maximization Algorithm
}

National Cancer Institute

\section{Source}

National Cancer Institute. Expectation Maximization Algorithm. NCI Thesaurus. Code C75945.

Algorithm used in statistics for finding maximum likelihood estimates of parameters in probabilistic models, where the model depends on unobserved latent (inferred) variables. 